\title{
GOLPH3 Overexpression is Closely Correlated with Poor Prognosis in Human Non-Small Cell Lung Cancer and Mediates its Metastasis Through Upregulating MMP-2 and MMP-9
}

\author{
Ran Wang Zun-fu Ke Fen Wang Wen-hui Zhang Yue-feng Wang Shu-hua Li
} Lian-tang Wang

Department of Pathology, First Affiliated Hospital of Sun Yat-sen University, Guangzhou, Guangdong, People's Republic of China

\section{Key Words}

$\mathrm{NSCLC} \cdot \mathrm{GOLPH} 3 \cdot$ Prognosis $\bullet$ Metastasis $\bullet \mathrm{MMP}-2 \cdot \mathrm{MMP}-9$

\begin{abstract}
Background/Aims: Golgi phosphoprotein 3 (GOLPH3) is a newly reported oncogene that plays a significant role in regulating cell growth. Recent research has shown that overexpression of GOLPH3 is correlated with patient survival and $\mathrm{M}$ classification in breast cancer and other cancers. However, the mechanisms by which GOLPH3 contributes to metastasis in non-small cell lung cancer (NSCLC) have not been previously clarified and are therefore the focus of this work. Methods: Immunohistochemistry (IHC) and western blotting analysis were performed to assess the GOLPH3 protein level, small interfering RNA (siRNA) and transwell assays were conducted to investigate the role of GOLPH3 in migration and invasion, and real-time PCR was performed to estimate the level of GOLPH3 mRNA expression. Results: GOLPH3 was significantly correlated with clinicopathological variables, such as the clinical stage $(P=0.012)$, T classification ( $P=0.002)$ and metastasis (M classification) $(P=0.008)$, in NSCLC patients and was negatively correlated with the prognosis. Knockdown of GOLPH3 significantly suppressed the migratory and invasive ability of NSCLC cell lines and downregulated the enzyme activity and protein levels of MMP-2 and MMP-9. Conclusions: The expression level of GOLPH3 is correlated with metastasis and prognosis in NSCLC, and GOLPH3 mediates metastasis by regulating the protein levels of MMP-2 and MMP-9 in vitro.

R. Wang, Z.-f. Ke and F. Wang contributed equally to this work.

Dr. Lian-Tang Wang

KARGER 125 


\section{Introduction}

According to the global oncology statistics for 2008, lung cancer is the most frequent malignancy in the world [1]. Lung cancer accounts for approximately $13 \%$ of cancer diagnoses, and $80 \%-85 \%$ are non-small cell lung cancer (NSCLC) cases [2]. The leading cause of death in lung cancer is primary tumor metastasis, particularly distant metastasis. In recent years, many newly identified oncogenes, such as astrocyte elevated gene-1 (AEG1 ), have been shown to play a significant role in lung cancer metastasis [3]. However, there is still no effective therapeutic target protein in patients with metastatic lung cancer, making it necessary to identify novel specific targets for treating patients with metastatic lung cancer.

Golgi phosphoprotein 3 (GOLPH3) is a highly conserved protein with a molecular weight of $34 \mathrm{kDa}[4,5]$ that is predominantly located in the external membrane of the trans-face of the Golgi, and it was initially identified through a proteomics analysis of the Golgi apparatus [4]. In 2009, Scott et al. first reported the overexpression of GOLPH3 in cancer [6]. Subsequently, amplification of the GOLPH3 gene was detected in 56.25\% of lung cancers, $38 \%$ of ovarian cancers, $37 \%$ of prostate cancers and $32 \%$ of gliomas and colon adenocarcinomas [6]. Further studies showed that GOLPH3 can activate mammalian target of rapamycin (mTOR) signaling pathway and sensitize the tumor cells to rapamycin and regulates cell growth, proliferation, colony formation, and tumor growth in many types of cancer, including prostate cancer [7], breast cancer [8] and glioma [6, 9, 10]. It was also reported that GOLPH3 expression is related to the M classification for metastasis and overall survival in patients with breast cancer[8] and prostate cancer [7]. Zhou et al. also reported that GOLPH3 regulates the invasion and migration of glioma through RhoA [11]. These investigations indicate that GOLPH3 plays an important role in tumor initiation and progression. Although the clinical role of GOLPH3 in NSCLC has been described $[12,13]$, the role of GOLPH3 in NSCLC metastasis and its mechanism remains unknown. In the present study, we focus on the role of GOLPH3 in the metastasis of NSCLC cells and investigate the underlying mechanism of its action.

\section{Materials and Methods}

\section{Cell lines and cell culture}

The NSCLC cell lines used in this study were obtained from the cell bank of our laboratory, and the human lung fibroblast cell line HLF was kindly provided by Professor Can-mao Xie (First Affiliated Hospital of Sun Yat-sen University). L78, A549, SPL-A1, and GLC-82 cells were cultured in RPMI 1640 medium (Gibco) supplemented with 10\% fetal bovine serum (FBS) (Bioind). The HLF cell line was cultured in DMEM medium (Gibco) containing 10\% fetal bovine serum. All growth media contained $100 \mu \mathrm{g} / \mathrm{L}$ streptomycin and $100 \mathrm{U} / \mathrm{L}$ penicillin. The cells were maintained at $37^{\circ} \mathrm{C}$ in a humidified incubator with $95 \%$ air and $5 \%$ $\mathrm{CO}_{2}$.

Patient characteristics and cancer specimens

Between January 2002 and December 2007, 230 specimens from patients diagnosed with NSCLC were collected at the First Affiliated Hospital of Sun Yat-sen University, and 146 of these had available followup data. Additionally, 18 pairs of tumor samples and paraneoplastic tissues were obtained from surgical specimens of patients who were diagnosed with NSCLC from March 2012 to June 2012. None of the patients had undergone radiotherapy or chemotherapy before surgery. The histological characteristics and clinical stages of the NSCLC patients were determined according to the criteria of the World Health Organization. Prior patient consent and approval from the Institutional Research Ethics Committee for the use of this clinical material for research purposes were obtained.

Western blotting

All tissues and cells were lysed with RIPA lysis buffer (1\% NP-40, 0.5\% sodium deoxycholate, $0.1 \%$ SDS in $1 \times$ PBS) containing PMSF and protease inhibitor (Roche) and then sonicated before centrifugation $(14,000 \times \mathrm{g})$ at $4^{\circ} \mathrm{C}$ for 15 minutes. The protein was quantitated using a BCA protein assay kit (ComWin 
Wang et al.: High Expression of GOLPH3 in Non-Small Cell Lung Cancer Correlates with Poor Prognosis and Metastasis

Biotech), separated on $12.5 \%$ SDS-PAGE gels, and transferred to PVDF membranes. Membranes were blocked with $5 \%$ non-fat milk, incubated with the indicated primary antibodies and then incubated with the corresponding horseradish peroxidase-conjugated secondary antibodies. The antibodies used included rabbit anti-human GOLPH3 (LifeSpan), rabbit anti-human GAPDH (Santa Cruz), mouse anti-human MMP2 (Abgent), and mouse anti-human MMP-9 (Abgent), rabbit anti-human phosphor-S6K and S6K (Cell Signaling Technology) primary antibodies and goat anti-rabbit and anti-mouse secondary antibodies (Cell Signaling Technology). The protein bands were visualized using an enhanced chemiluminescence (ECL) kit (Millipore).

\section{Immunohistochemical staining}

Immunohistochemical (IHC) staining was used to detect the protein expression in paraffin-embedded tissues. The sections were immersed in sodium citrate repair solution $(\mathrm{pH} \mathrm{6.0)}$ under high pressure for 3 minutes, soaked in $3 \% \mathrm{H}_{2} \mathrm{O}_{2}$ for 30 minutes, and then blocked with normal goat serum at $37^{\circ} \mathrm{C}$ for 1 hour. Rabbit anti-human GOLPH3, mouse anti-human MMP-2, and rabbit anti-human MMP-9 antibodies were used for staining. After overnight incubation at $4^{\circ} \mathrm{C}$, the slides were incubated with general immunohistochemical secondary antibodies (DAKO) for 30 minutes at $37^{\circ} \mathrm{C}$ and then visualized with DAB. The immunoreactivity scores of the cancer tissue samples were determined based on the staining intensity and area of positive staining according to the method described in Sun et al. [3]. Two pathologists evaluated all slides. A total score $\geq 6$ was defined as high expression, and a score $<6$ was defined as low expression.

\section{Real-time PCR analyses}

Total RNA was extracted using TRIzol (Roche) in accordance with the manufacturer's instructions, and mRNA was reverse transcribed to cDNA using a reverse transcription regent kit (Fermentas). The cDNA concentration was measured using a mini spectrophotometer (ABI). PCR was performed using the fluorescence-based quantitative SYBR GREEN kit (Roche). The primer sequences used for each target gene were as follows:

GOLPH3,

forward: 5'-TCCTGGAGAATGCTTTTGCT-3', reverse: 5'-GGTGTTGGCCTTCAGACATT-3';

GAPDH,

forward: 5'-GTGGACCTGACCTGCCGTCT-3', reverse: 5'-GGAGGAGTGGGTGTCGCTGT-3';

MMP-2,

forward: 5'-CTGGGAGCATGGCGATGGATA-3', reverse: 5'-GGAAGCGGAATGGAAACTTG-3';

MMP-9,

forward: 5'-GCCATGTCTGCTGTTTTCTAGAGG-3', reverse: 5'-CACACTCCAGGCTCTGTCCTCTTT-3'.

The cycling reaction was as follows: $95^{\circ} \mathrm{C}$ for 10 minutes, followed by 40 cycles of $95^{\circ} \mathrm{C}$ for 15 seconds and $62^{\circ} \mathrm{C}$ for 1 minute. The conditions for the melting curve analysis were $95^{\circ} \mathrm{C}$ for 15 seconds and $60^{\circ} \mathrm{C}$ for 1 minute, which was followed by an increase in temperature to $95^{\circ} \mathrm{C}$ over 15 seconds at a rate of $1 \%$. The gene expression levels were analyzed using the $-\Delta \Delta \mathrm{Ct}$ method.

\section{Transient interference assays}

Small interfering RNA (siRNA) technology was used to silence the expression of the target gene GOLPH3 in cell lines. Approximately $2 \times 10^{5}$ cells/well were seeded in 6-well plates and grown to a cell density of approximately 40\%; then, the medium was replaced with serum-free RPMI 1640 . A mixture of siRNA and Lipofectamine RNAiMAX (Invitrogen) was added to the cells according to the manufacturer's instructions. After 6 hours of incubation, the cells were cultured in RPMI 1640 medium supplemented with $10 \%$ FBS. RNA and protein were extracted after transfection. The siRNA sequences used in this study were as follows:

GOLPH3,

forward, 5'-GAAUUAGCAUUGAGAGGAA-3'

antisense, 3'-CUUAAUCGUAACUCUCCUU-5'; 
Wang et al.: High Expression of GOLPH3 in Non-Small Cell Lung Cancer Correlates with Poor Prognosis and Metastasis

control siRNA, forward, 5'-UUCUCCGAACGUGUCACGUTT-3'

antisense, 5'-ACGUGACACGUUCGGAGAATT-3' (Ribobio).

\section{Invasion and migration assay}

The invasion ability of cells was analyzed using Matrige $^{\mathrm{TM}}$ (BD) and Transwell@ (Corning) assays. Briefly, $60 \mu \mathrm{L}$ of a mixture of Matrigel ${ }^{\mathrm{TM}}$ and serum-free RPMI 1640 (at a ratio of 1:8) was added to the upper chamber of the Transwell ${ }^{\circledR}$, which was then placed in an incubator until the water evaporated. The Matrigel $^{\mathrm{TM}}$ was hydrated using basic RPMI 1640 for 30 minutes before the addition of cells. L78 and A549 cells were transfected with siRNA for 48 hours and resuspended at a density of $2 \times 10^{5} / \mathrm{mL}$ in serum-free RPMI-1640 medium. Approximately $2 \times 10^{4}$ cells/well were seeded in the upper chamber, and the lower well was filled with $700 \mu \mathrm{L}$ RPMI 1640 supplemented with $10 \%$ FBS. After 36 hours at $37^{\circ} \mathrm{C}$, any cells remaining in the upper chamber were removed. The cells in the lower chamber were fixed with $4 \%$ paraformaldehyde and stained with crystal violet, and the invasion ability was evaluated by counting the cells that had migrated to the lower side of the filter under a microscope at $400 \times$ magnification. Five fields were randomly selected for analysis.

The procedure for the migration assay was the same as above, except that the Transwell $®$ upper chamber did not contain Matrige ${ }^{\mathrm{TM}}$ and the incubation time was 24 hours.

\section{Isolation of invasive and noninvasive cell sublines using transwell chambers}

Sublines with high or low invasion and migration potential were isolated from the A549 cell line using 24-well plates coated with or without Matrigel ${ }^{\mathrm{TM}}$ prepared according to the protocol described above. Approximately $1 \times 10^{5}$ A549 cells were seeded in each insert of the Transwell $®$ and incubated for 48 hours. After incubation, the invasive/migratory cells on the lower side of the filter and the noninvasive/ nonmigratory cells in the upper chambers were harvested aseptically and expanded for next-round selection. After five rounds of selection, the sublines were designated as low-inv-A549 or high-inv-A549 and low-mig-A549 or high-mig-A549, depending on their ability to invade or migrate through the filter.

\section{Zymography}

After transfection with siRNA for 24 hours, the cells were cultured for 24 hours in serum-free RPMI 1640 medium and the conditioned medium was collected and centrifuged to obtain the supernatant. The protein was enriched using ultrafiltration (Millipore) at $4000 \mathrm{rpm}$ for 30 minutes before measuring the protein concentration with a BCA kit. For each sample, $30 \mu \mathrm{g}$ of protein was mixed with non-reducing loading buffer and separated by electrophoresis on a 10\% SDS-polyacrylamide gel (SDS-PAGE) containing $1 \%$ gelatin at $4^{\circ} \mathrm{C}$. After electrophoresis, the gel was processed according to the instructions for the gelatin zymography reagent (Applygen) until clear digested bands appeared; the gel was then photographically scanned.

\section{Statistics}

All data were analyzed using SPSS13.0 statistical software, and $P<0.05$ was considered statistically significant; plotting and image processing were performed with GraphPad Prism5 and Photoshop 7.0 software, respectively. A two-tailed t-test was used to analyze the data from real-time PCR and the invasion or migration assays. The chi-square test and Fisher's exact test were used to analyze the relationship between GOLPH3 expression and the clinicopathological characteristics. The bivariate correlations between study variables were calculated by Spearman's rank correlation coefficients. The survival data were analyzed with the Kaplan-Meier method and log-rank test.

\section{Results}

Expression of GOLPH3 in NSCLC cell lines and tissues

GOLPH3 expression in NSCLC cell lines and cancer tissues was examined by western blot analysis. As shown in Fig. 1A, GOLPH3 was highly expressed in all NSCLC cell lines tested, including the squamous cell line L78 and the adenocarcinoma cell lines A549, GLC-82, and 


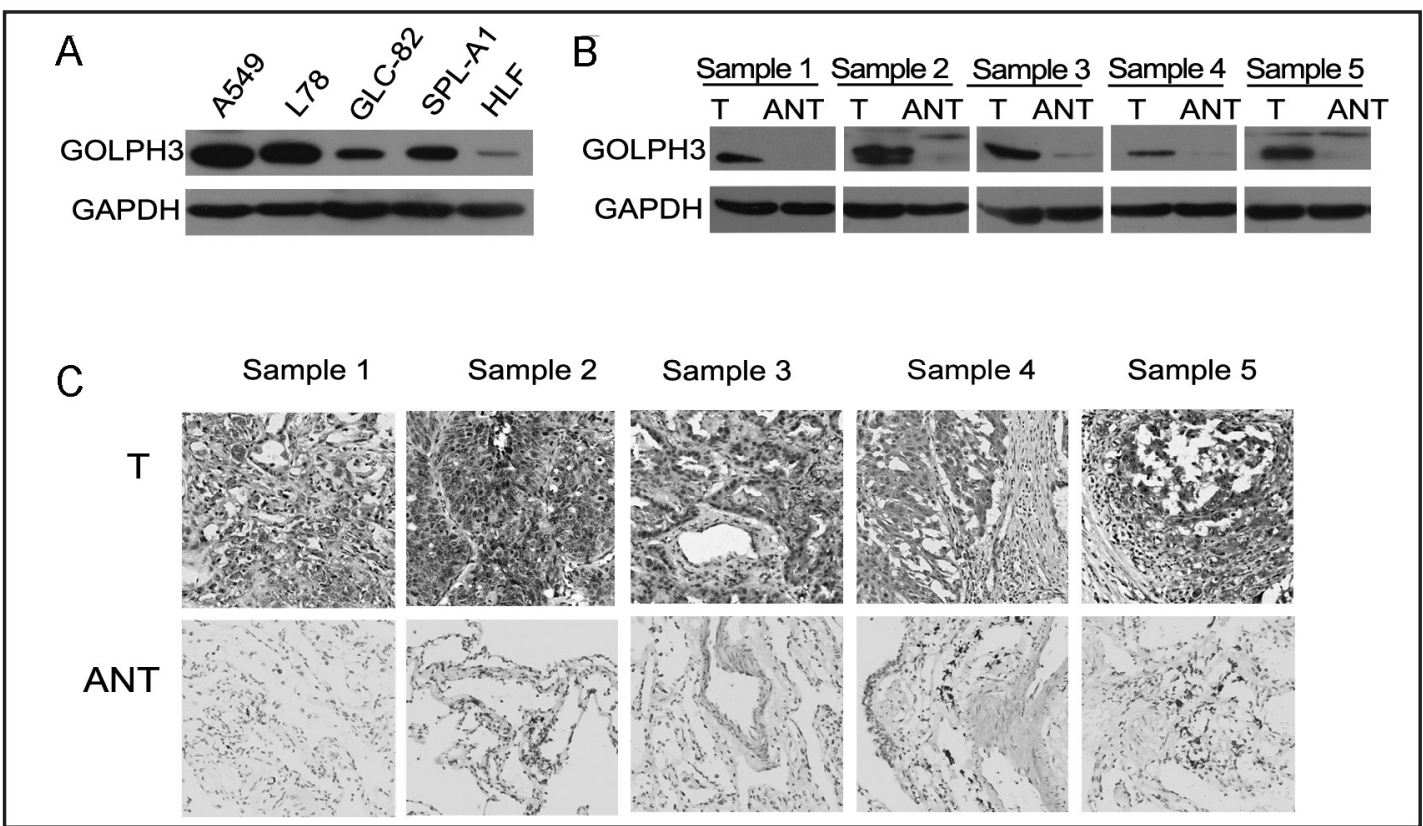

Fig. 1. GOLPH3 was highly expressed in NSCLC cell lines and primary NSCLC tissues. (A) The expression of the GOLPH3 protein in the NSCLC cell lines A549, GLC-82, SPL-A1, and L78 and the human lung fibroblast cell line HLF was detected by western blot analysis. GAPDH was included as a loading control. (B) The expression of GOLPH3 proteins in representative primary NSCLC cancer tissues (T) and adjacent normal tissues (ANT) was detected by western blot. GAPDH was used as an internal control. (C) Immunohistochemical staining of GOLPH3 protein in five representative cases of primary NSCLC (T) and adjacent normal tissues (ANT). The same samples were used in panels B and C. Original magnification $\times 100$.

SPL-A1. In contrast, GOLPH3 was weakly expressed in the human lung fibroblast cell line HLF. In addition, we performed western blot analysis of 19 pairs of matched surgically resected frozen tumor tissues and the adjacent lung tissues, including 11 cases of adenocarcinoma, 7 cases of squamous cell carcinoma, and 1 case of atypical epithelial hyperplasia. Compared with the low or absent expression in paraneoplastic tissues, GOLPH3 was highly expressed in matched tumor tissue from the same patient (Fig. 1B). The fresh frozen tissues were also embedded in paraffin for evaluation of the GOLPH3 expression by IHC staining. Consistent with the western blot analysis, the staining intensity was higher and the positive area was larger in cancer tissues compared with paracancerous tissues (Fig. 1C). These results suggest that GOLPH3 is highly expressed in NSCLC cells and tissues at the protein level.

\section{Overexpression of GOLPH3 in NSCLC tissues correlated with poor prognosis and distant} metastasis

To further examine the relationship between GOLPH3 expression and the clinicopathological parameters of NSCLC, 230 paraffin-embedded NSCLC tissues were evaluated by IHC staining, including 103 stage I cases, 57 stage II cases, 50 stage III cases, and 20 stage IV cases. Among these, 62.61\% (144 out of 230) showed high expression of GOLPH3. The relationship between GOLPH3 and clinicopathological parameters is summarized in Table 1. The expression level of GOLPH3 was significantly associated with the clinical stage, tumor size (T classification), distant metastases (M classification), and body mass index (BMI). The Spearman's correlation coefficients of GOLPH3 with the clinical stage, T classification, and M classification were $0.165(P=0.012), 0.202(P=0.002)$, and $0.176(P=0.008)$, respectively (Table 2$)$. However, GOLPH3 expression was not related to age, gender, histological type, differentiation, node (N classification), or smoking history. As shown in Fig. 2A, the expression of GOLPH3 was significantly increased in patients with stages III and IV disease, particularly in those with distant metastasis. A survival analysis 


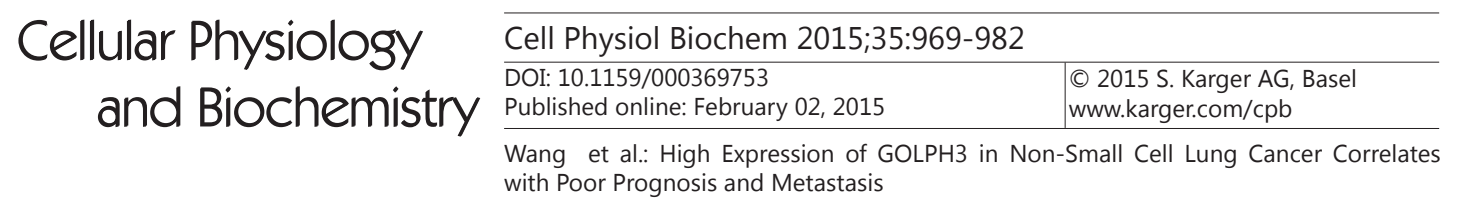

Table 1. Correlation between GOLPH3 protein expression and the clinicopathologic characteristics of NSCLC patients

\begin{tabular}{|c|c|c|c|c|}
\hline \multirow[t]{2}{*}{ Characteristics } & \multicolumn{2}{|c|}{ GOLPH3 } & \multirow{2}{*}{$\begin{array}{l}\text { Chi-square } \\
P \text {-value }\end{array}$} & \multirow{2}{*}{$\begin{array}{l}\text { Fisher's exact test } \\
P \text {-value }\end{array}$} \\
\hline & $\begin{array}{l}\text { Low expression } \\
\text { no.(\%) }\end{array}$ & $\begin{array}{l}\text { High expression } \\
\text { no.(\%) }\end{array}$ & & \\
\hline \multicolumn{5}{|l|}{ Gender } \\
\hline Male & $61(70.93)$ & $102(70.83)$ & 0.998 & 1.000 \\
\hline Female & $25(29.07)$ & $42(29.17)$ & & \\
\hline \multicolumn{5}{|l|}{ Age(years) } \\
\hline$\leq 65$ & $49(56.98)$ & $96(66.67)$ & 0.141 & 0.159 \\
\hline$>65$ & $37(43.02)$ & $48(33.33)$ & & \\
\hline \multicolumn{5}{|l|}{ Clinical stage } \\
\hline I & $43(50.00)$ & $60(41.67)$ & 0.004 & 0.003 \\
\hline II & $28(32.56)$ & $29(20.14)$ & & \\
\hline III & $13(15.12)$ & $37(25.69)$ & & \\
\hline IV & $2(2.33)$ & $18(12.50)$ & & \\
\hline \multicolumn{5}{|l|}{$\mathrm{T}$ classification } \\
\hline T1 & $41(47.67)$ & $45(31.25)$ & 0.10 & 0.008 \\
\hline T2 & $35(40.70)$ & $63(43.75)$ & & \\
\hline T3 & $8(9.30)$ & $17(11.81)$ & & \\
\hline $\mathrm{T} 4$ & $2(2.33)$ & $19(13.19)$ & & \\
\hline \multicolumn{5}{|l|}{$\mathrm{N}$ classification } \\
\hline $\mathrm{Nx}$ & $1(1.16)$ & $8(5.56)$ & 0.138 & 0.143 \\
\hline No & $54(62.79)$ & $82(56.94)$ & & \\
\hline N1 & $17(19.77)$ & $20(13.89)$ & & \\
\hline N2 & $14(16.280$ & $34(23.61)$ & & \\
\hline \multicolumn{5}{|l|}{ M classification } \\
\hline Mo & $83(96.51)$ & $123(85.42)$ & 0.008 & 0.007 \\
\hline M1 & $3(3.49)$ & $21(14.58)$ & & \\
\hline \multicolumn{5}{|l|}{ Histology } \\
\hline Adenocarcinoma & $46(53.49)$ & $88(61.11)$ & 0.224 & 0.228 \\
\hline Squamous & $36(41.86)$ & $54(37.50)$ & & \\
\hline Other & $4(4.65)$ & $2(1.39)$ & & \\
\hline \multicolumn{5}{|c|}{ Histology differentiation } \\
\hline Well & $7(8.14)$ & $5(5.34)$ & 0.297 & 0.323 \\
\hline Moderate & $43(50.00)$ & $78(54.17)$ & & \\
\hline Poor & $36(41.86)$ & $61(42.36)$ & & \\
\hline \multicolumn{5}{|l|}{ Smoking history } \\
\hline No & $45(52.33)$ & $85(59.03)$ & 0.321 & 0.339 \\
\hline Yes & $41(47.67)$ & $59(40.97)$ & & \\
\hline \multicolumn{5}{|l|}{ BMI } \\
\hline$\leq 20$ & $3(3.49)$ & $10(6.94)$ & 0.025 & 0.024 \\
\hline$\leq 25$ & $71(82.56)$ & $95(65.97)$ & & \\
\hline$>25$ & $12(13.96)$ & $39(27.08)$ & & \\
\hline
\end{tabular}

Table 2. Correlation of GOLPH3 expression and clinical stage, $\mathrm{T}$ and M classification in NSCLC patients

\begin{tabular}{lcc}
\hline Variables & GOLPH3 expression level Spearman Correlation & $P$-value \\
\hline Clinical stage & 0.165 & 0.012 \\
T classification & 0.202 & 0.002 \\
M classification & 0.176 & 0.008 \\
\hline
\end{tabular}

indicated an inverse correlation between the GOLPH3 expression level and the overall survival time of patients with NSCLC; patients with a high expression level usually had a shorter survival time (Fig. 2B). Taken together, these data indicate that GOLPH3 can be used to predict distant metastasis and prognosis in patients with NSCLC. In addition, IHC staining 
A
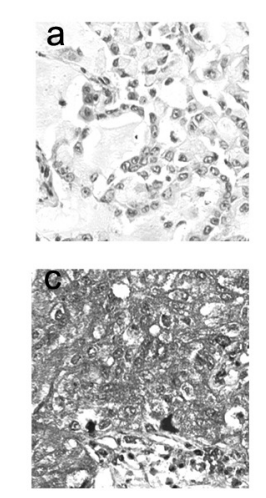

C
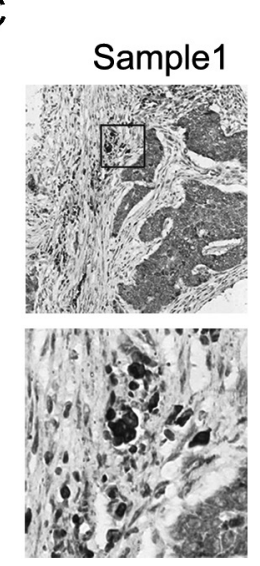

B
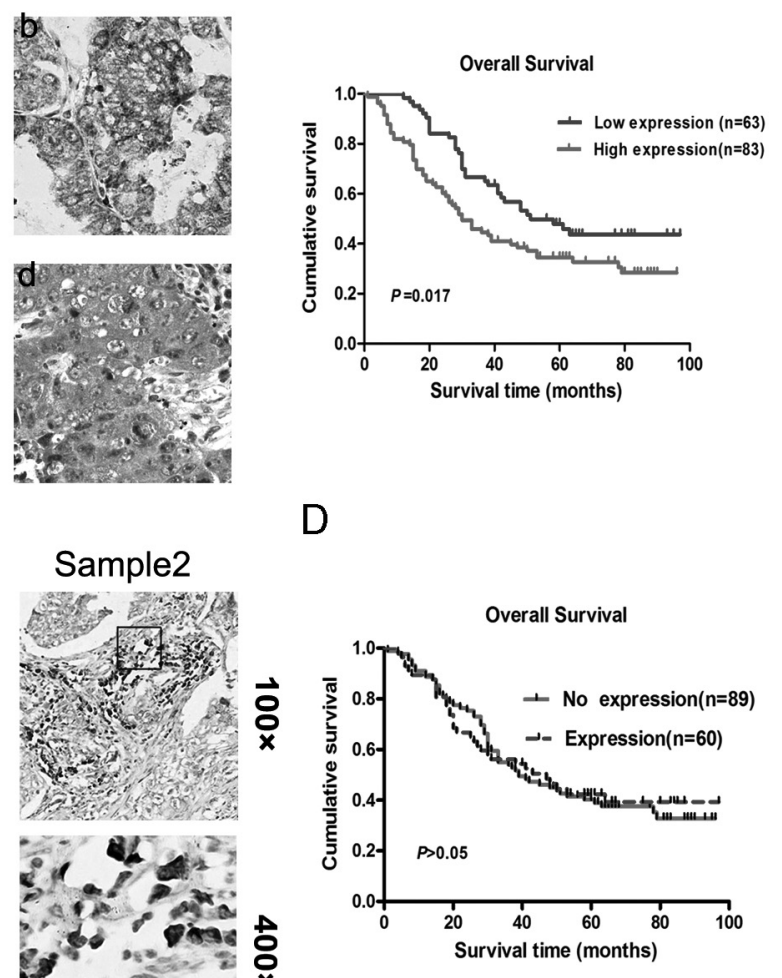

$\mathrm{D}$

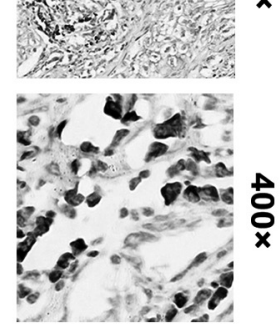

Fig. 2.The expression of GOLPH3 was elevated in paraffin-embedded NSCLC sections, and its expression level was closely correlated with the prognosis in patients with NSCLC. (A) Representative images of IHC staining with an anti-GOLPH3 antibody in paraffin-embedded tumor samples from patients with NSCLC at different clinical stages (a, stage I; b, stage II; c, stage III; and d, stage IV). Original magnification $\times 200$. (B) Correlation between the GOLPH3 protein expression and the survival of NSCLC patients $(P=0.017$, log-rank test). (C) The expression of GOLPH3 proteins was detected in the stroma of NSCLC tissues by IHC. Original magnification $\times 200$ (upper) and $\times 400$ (lower). (D) Kaplan-Meier survival curves with univariate analyses (log-rank) for patients who were negative or positive for GOLPH3 expression in the tumor stroma. There was no significant difference between the groups with or without GOLPH3 expression in cancer stroma $(P$ $>0.05)$.

demonstrated that GOLPH3 was also expressed in the tumor stroma (Fig. 2C). However, in contrast to the expression of GOLPH3 in tumor cells, the expression of GOLPH3 in the stroma was not correlated with the survival time (Fig. 2D) or clinicopathological parameters.

Overexpression of GOLPH3 was closely related to the invasiveness of NSCLC cells in vitro

To further investigate the biological role of GOLPH3 in the process of NSCLC metastasis, migration and invasion assays were performed. As shown in Fig. 3B and 3C, silencing of GOLPH3 expression inhibited the invasion and migration of A549 and L78 cells, as evidenced by the migration of fewer cells to the lower side of the filter. To further explore the role of GOLPH3 in invasion and migration, we established high-invasion/migration (high-inv-A549 and high-mig-A549) and low-invasion/migration (low-inv-A549 and low-mig-A549) sublines from the A549 NSCLC cell line using repeated transwell assays. The paired cell sublines had the same genetic background and were phenotypically identical except for their invasive/ migratory ability. When we analyzed the invasion and migration ability of these sublines, invasion of the high-inv-A549 cells was approximately three-fold higher than that of low- 


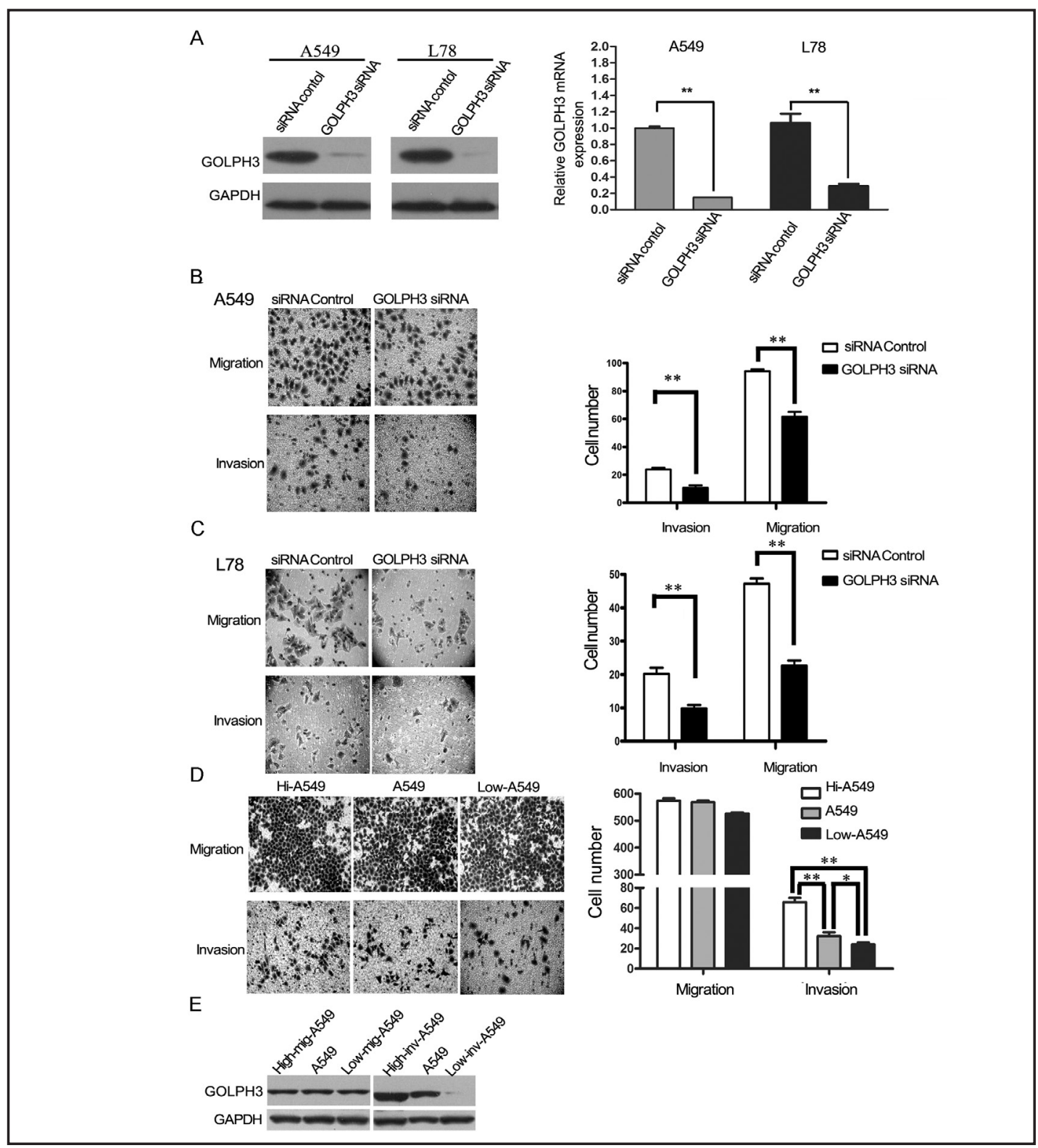

Fig. 3. Downregulation of GOLPH3 inhibited the invasion and migration ability of A549 and L78 cells. (A) Downregulation of GOLPH3 by siRNA in A549 and L78 cells was confirmed by western blot (left) and real-time PCR (right). ${ }^{*}, P<0.05$. GAPDH was included as a loading control. (B) Silencing of GOLPH3 by siRNA inhibited the migration and invasion ability of A549 cells (left), as confirmed by quantification of the migrated and invaded cells on the lower side of the filter (right). ${ }^{* *}, P<0.01$. Magnification $\times 200$. (C) Silencing of GOLPH3 by siRNA inhibited the migration and invasion of L78 cells (left), as confirmed by quantification of the migrated and invaded cells on the lower side of the filter (right). ${ }^{* *}, P<0.01$. Magnification $\times 200$. (D) The established high-invasion/migration and low-invasion/migration cell sublines were characterized using a Transwell@ assay (left); the bar graph represents the average number of cells on the lower side of the filter (right). ${ }^{*}, P<0.05 ;{ }^{* *}, P<0.01$. Magnification $\times 200$. (E) The expression of GOLPH3 in different A549 cell sublines was detected by western blot. GAPDH was used as a loading control. The error bars (SD) were calculated from triplicate experiments.

inv-A549 cells, as shown in Fig. 3D, whereas there was very little difference in the migration between the high-mig-A549 and low-mig-A549 cells. As shown in Fig. 3E, western blot analysis revealed that the expression of GOLPH3 protein was upregulated in high-inv-A549 
Fig. 4. Downregulation of GOLPH3 with siRNA inhibited NSCLC cell migration and invasion through the regulation of MMP-2 and MMP-9. (A) Downregulation of GOLPH3 by siRNA reduced the protein levels of MMP-2 and MMP-9 in A549 and $\mathrm{L78}$ cells. GAPDH was used as a loading control. (B) The expression level of GOLPH3 protein correlated with the MMP-2 and MMP9 expression in A549 sublines. GAPDH was used as a loading control. (C) Downregulation of GOLPH3 by siRNA reduced the gelatinolytic activity in A549 and L78 cells. (D) Representative IHC staining of GOLPH3, MMP-2, and MMP-9 expression in paired, paraffin-embedded NSCLC tissues. Original magnification $\times 200$.
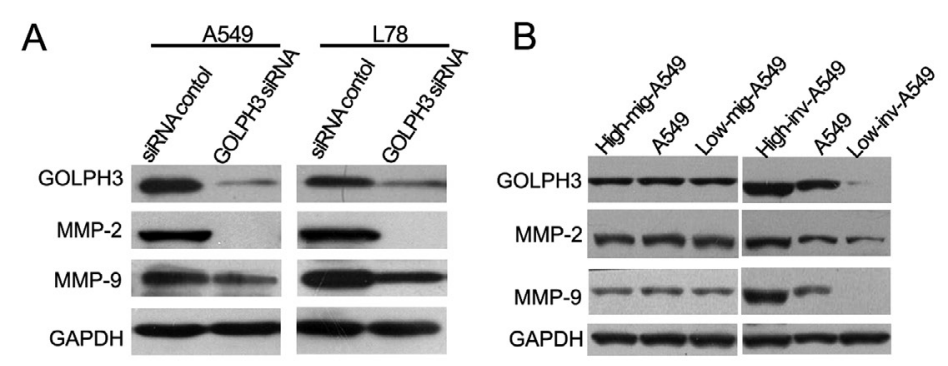

C

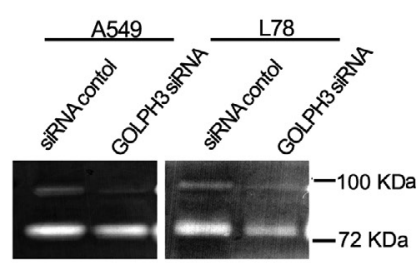

$\mathrm{D}$

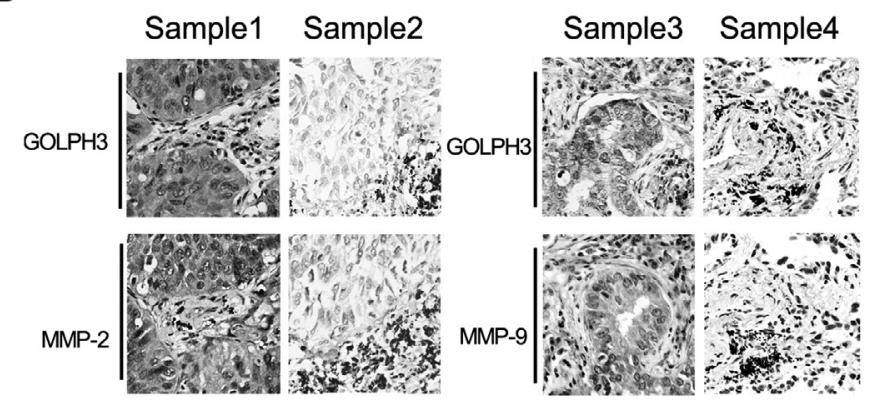

Table 3. Correlation of GOLPH3 and MMP-2, MMP-9 in NSCLC patients

\begin{tabular}{lcc}
\hline Variables & GOLPH3 expression level Spearman Correlation & $P$-value \\
\hline MMP-2 & 0.223 & 0.023 \\
MMP-9 & 0.251 & 0.01 \\
\hline
\end{tabular}

and downregulated in the low-inv-A549 subline compared with the parental A549 cells; however, there was no difference between the high-mig-A549, A549, and low-mig-A549 cells. These results suggest that GOLPH3 regulates the invasion and migration abilities of L78 and A549 cells in vitro.

GOLPH3 modulated the invasion of NSCLC cell lines by regulating MMP-2 and MMP-9

MMP-2 and MMP-9, the most well-studied members of the matrix metalloproteinase (MMP) family, play a leading role in tumor invasion and metastasis. We therefore investigated whether MMP-2 and MMP-9 play a role in cell invasion mediated by GOLPH3. As shown in Fig. 4A, downregulation of GOLPH3 by siRNA silencing was associated with decreased protein levels of MMP-2 and MMP-9. Furthermore, western blot analysis of the expression of GOLPH3, MMP-2, and MMP-9 in high-inv-A549, low-inv-A549, high-mig-A549, and lowmig-A549 cells revealed that the expression levels of MMP-2 and MMP-9 were much higher in high-inv-A549 than in low-inv-A549 cells, whereas there was almost no difference in the expression among high-mig-A549, A549, and low-mig-A549 cells (Fig. 4B). We next analyzed 
Wang et al.: High Expression of GOLPH3 in Non-Small Cell Lung Cancer Correlates with Poor Prognosis and Metastasis

Fig. 5. Downregulation of GOLPH3 increased the degradation and decreased the translation of MMP-2 and MMP9. (A)The relative mRNA expression level of GOLPH3, MMP-2 and MMP-9 was measured by real-time PCR in A549 and L78 cells after GOLPH3 silencing by siRNA. * $P<0.05$; **, $P<0.01$. GAPDH was used as an internal control. The error bars (SD) were calculated from triplicate experiments. (B)The expression of MMP-2 and MMP9 in A549 and L78 was analyzed by western blot after treating the cell lines with the proteasome inhibitor MG-132 (5 $\mu \mathrm{mol} / \mathrm{L})$ and GOLPH3 siRNA. GAPDH was used as a loading control. (C)The expression of MMP2 and GOLPH3 in A549 was evaluated by western blot in different groups, including siRNA Control, siRNA Control + CHX and GOLPH3 SiRNA + CHX. (D)Ex-

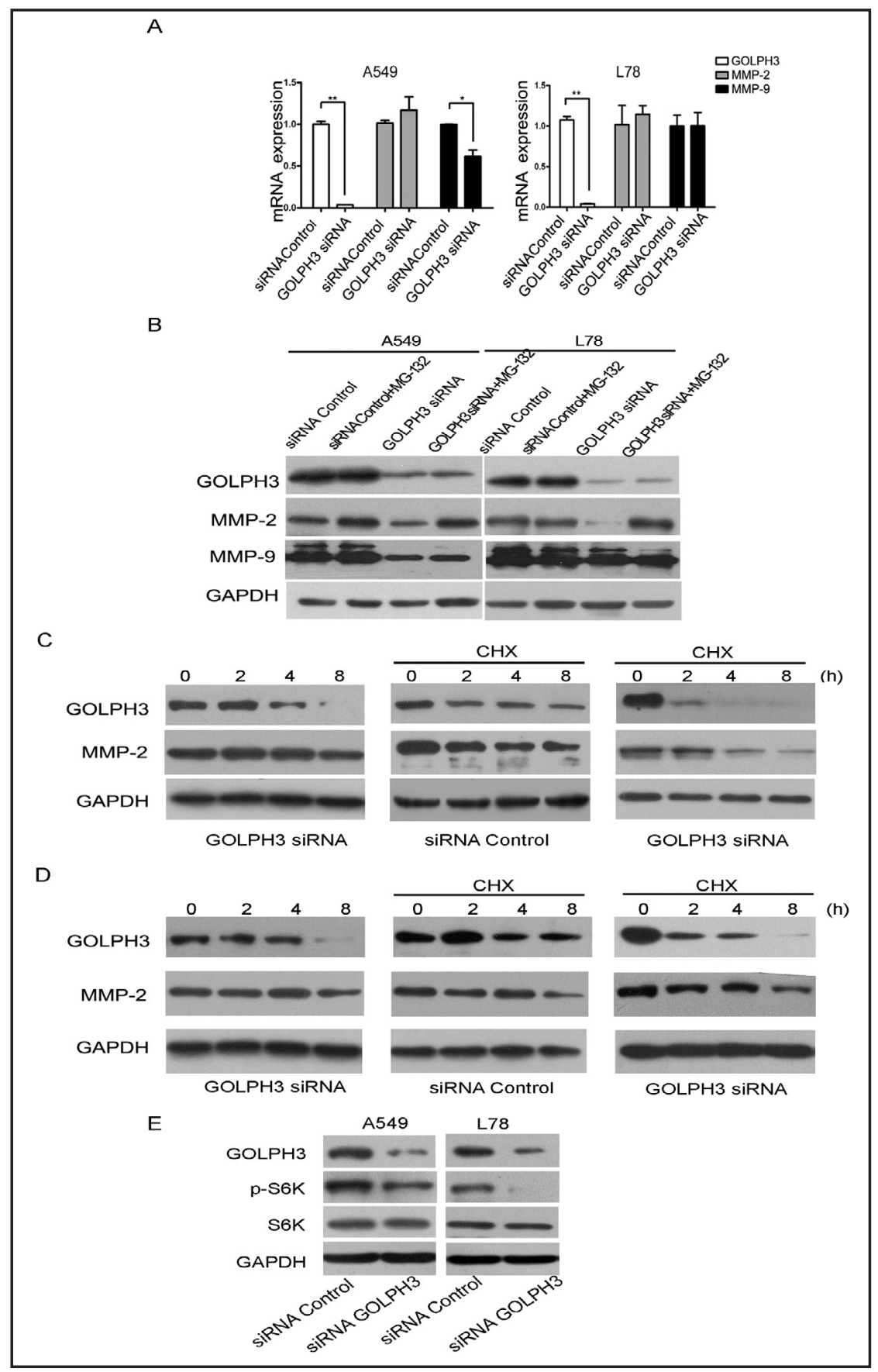

pression of MMP-2 and GOLPH3 in L78 cells was evaluated by western blot in different groups, including siRNA Control, siRNA Control + CHX and GOLPH3 siRNA + CHX. (E)The expression of the indicated proteins was detected by western blot analysis of A549 and L78 cells after GOLPH3 silencing.

the effect of GOLPH3 on the gelatinolytic activity of MMPs using a gelatin zymography assay and found that the gelatinolytic activity of MMP-2 and MMP-9 was reduced after the silencing of GOLPH3 expression (Fig. 4C). These results indicate that GOLPH3 regulates both the expression and the gelatinolytic activity of MMP-2 and MMP-9.

To confirm the relationship between the expression of GOLPH3 and that of MMP-2 and MMP-9 in NSCLC tissues, we measured the expression of GOLPH3, MMP-2, and MMP9 in NSCLC tissues by IHC. As shown in Fig. 4D and Table 3, tissues with high GOLPH3 expression typically displayed high levels of MMP-2 and MMP-9 with Spearman's correlation 
coefficients of $0.223(P=0.023)$ and $0.251(P=0.01)$, respectively. These results indicate that the expression of GOLPH3 is positively correlated with the MMP-2 and MMP-9 levels in NSCLC tissues.

GOLPH3 downregulation increased the degradation of MMP-2 and MMP-9 in A549 and L78 cells

To explore the mechanism by which GOLPH3 regulated the expression of MMP-2 and MMP-9, we used a real-time quantitative PCR assay to evaluate the MMP-2 and MMP-9 mRNA levels in cells treated with GOLPH3 siRNA. After transfection for 48 hours, the MMP-2 mRNA levels in A549 and L78 cells and the MMP-9 mRNA levels in L78 cells were unchanged, but the MMP-9 mRNA level was downregulated in A549 cells (Fig. 5A). This decrease, induced by GOLPH3 in MMP-2 protein, might be due to a decrease in the translation or an increase in the proteasomal degradation of MMP-2. To elucidate the mechanism of regulation, the proteasome inhibitor MG-132 and the protein synthesis inhibitor cycloheximide (CHX) were used. A549 and L78 cells were first treated with the proteasome inhibitor MG-132, and the protein expression of MMP-2 and MMP-9 was detected by western blot (Fig. 5B). MG132 significantly inhibited the decrease in MMP-2 expression that was induced by GOLPH3 siRNA transfection in both A549 and L78, but it had a lower effect on MMP-9 expression in the A549 and L78 cell lines, particularly in L78 cells. To further elucidate the mechanism, we inhibited protein synthesis with CHX $(5 \mu \mathrm{mol} / \mathrm{L})$ for 2,4 , and 8 hours in the siRNA control group and the GOLPH3 siRNA group in both cell lines (Fig. 5C and Fig. 5D), and the protein level of GOLPH3 and MMP-2 was evaluated by western blot. MMP-2 was degraded faster in cells treated with GOLPH3 siRNA and CHX than in cells treated with the control siRNA and CHX, indicating that GOLPH3 downregulation can increase MMP-2 degradation through the proteasomal degradation pathway. Moreover, S6K, an important protein in the mTOR pathway, can affect protein synthesis by activating cap-dependent translation initiation, including the activation of eIF4E through the phosphorylation of 4EBP-1. Compared with the siRNA control group in A549 and L78 cells, GOLPH3 downregulation decreased the phosphorylation of S6K at thr359 (Fig. 5F), indicating that GOLPH3 may affect protein synthesis. All of these results suggest that GOLPH3 might modulate MMP-2 at the protein level by increasing its degradation and decreasing protein synthesis, and, to some extent, GOLPH3 affectes MMP-9 at the RNA level in A549 cells.

\section{Discussion}

GOLPH3, also known as GPP34, GMX33, or MIDAS [4, 5], is a phosphorylated protein located in the Golgi complex. Schmitz et al. previously found that deletion of VPS74, which is a homologue of GOLPH3 in budding yeast, led to defective transition and secretion of glycosylated protein [14], and the authors proposed that GOLPH3 affects glycosyl transferases in the Golgi complex and is therefore involved in protein glycosylation. As many oncogenes are glycosylated proteins that play a role in tumor cell growth, adhesion, migration, invasion, immune recognition, and signal transduction [15], GOLPH3 expression may be closely related to tumorigenesis [16]. Moreover, Scott et al. demonstrated that GOLPH3 can activate mTOR signaling [6], which is involved in the regulation of several biological behaviors of tumor cells, including cell growth, cell cycle, angiogenesis, invasion, and metastasis[17]. Therefore, it is speculated that GOLPH3 might play an important role in the occurrence and development of tumors. In 2009, Scott et al. reported that a FISH analysis demonstrated that the GOLPH3 gene was amplified in a variety of solid tumor tissues [6], and GOLPH3 was subsequently found to be overexpressed in many tumor tissues, including prostate cancer [7], breast cancer [8], and glioma [10]. It was also reported that the level of GOLPH3 expression was correlated with the M classification and with the overall survival of breast cancer [8], prostate cancer [7] and NSCLC patients [12,13]. These investigations indicated the significance of GOLPH3 in tumor initiation and progression in some types of cancer.

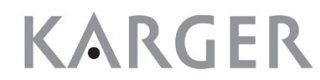


Wang et al.: High Expression of GOLPH3 in Non-Small Cell Lung Cancer Correlates with Poor Prognosis and Metastasis

In NSCLC tissues, the biological role of GOLPH3 is limited to cell proliferation, the colony forming ability, the regulation of rapamycin sensitivity [6], and high microvessel density [13]. The western blot results in our study demonstrated that GOLPH3 was highly expressed at the protein level in both NSCLC tissue sections and cell lines. As determined by IHC staining, 144 of 230 NSCLC tissue sections displayed moderate to strong staining of GOLPH3, whereas there was no significant GOLPH3 staining in the adjacent, non-cancerous tissue. Statistical analysis revealed that the expression level of GOLPH3 was correlated with the M classification and clinical stage, supporting the notion that GOLPH3 might play an important role in the progression of NSCLC, which was consistent with Zhang's study [12], whereas it was not in agreement with Lu's study reporting that GOLPH3 expression did not correlate with the clinicopathological features of early-stage NSCLC [13]. This inconsistency may be due to differences in the clinical stages of NSCLC; while there was no relationship between GOLPH3 expression and early-stage

NSCLC in Lu's study, there could still be a correlation between GOLPH3 expression and all stages of NSCLC. Moreover, patients with a high GOLPH3 expression level usually had a shorter survival time than patients with low expression. All of these findings suggest that GOLPH3 might be used as a predictor for disease progression and prognosis in patients with NSCLC.

The tumor microenvironment consists of fibroblasts, mesenchymal cells and immune cells, including macrophages, mast cells, and lymphocytes [18]. These cells can promote tumor growth and metastasis via cell signaling pathways activated by cytokines, and they can affect the patient survival [19]. GOLPH3 is believed to be involved in protein glycosylation and the regulation of protein secretion, such as the secretion of cytokines; therefore, we evaluated the GOLPH3 expression in the cells in the tumor stroma and attempted to elucidate the relationships between GOLPH3 expression and the patients' survival time as well as the clinicopathological parameters. Neither the NSCLC patient survival nor the clinicopathological parameters were correlated with GOLPH3 expression; however, elevated GOLPH3 expression in cancer cells plays a major role in regulating the biological behaviors of NSCLC cells, particularly metastasis.

We found that the expression level of GOLPH3 is closely related to the M classification in tissue sections, which is consistent with other studies [8, 12]. We also found that GOLPH3 can regulate the migration and invasion abilities of NSCLC cell lines, including A549 and L78; meanwhile, the invasion and migration of these cell lines was reduced when GOLPH3 was knocked down using siRNA. Moreover, compared with control A549 cells, a subline of A549 cells with high invasive ability that was established by repeated transwell experiments showed high expression of GOLPH3, whereas a cell line with low invasive ability had low expression. These results suggest that GOLPH3 expression is correlated with the invasiveness of cell lines and metastasis in NSCLC tissues.

Metastasis is a complex multistep process that includes degradation of the basement membrane and the extracellular matrix [20]. The MMP family of proteinases, particularly MMP-2 and MMP-9, plays an important role in these processes [20-22]. Therefore, we explored whether GOLPH3 mediated the metastasis of NSCLC through MMP-2 and MMP-9 and found that the expression of MMP-2 and MMP-9 was reduced after GOLPH3 silencing. Moreover, studies of A549 cell sublines (A549-high-invasion and A549-low-invasion) demonstrated that the expression of MMP-2 and MMP-9 was correlated with the expression of GOLPH3, which was further confirmed in this study with correlation coefficients for IHC staining of NSCLC tissue sections of $0.223(P=0.023)$ and $0.251(P=0.01)$, respectively.

As the proteolysis of gelatin by MMP proteins plays a vital role in collagen degradation and, therefore, metastasis, we studied the effect of GOLPH3 expression on gelatinolytic activity. In a zymography assay, the gelatinolytic activity of MMP-2 (shown at $72 \mathrm{kDa}$ ) and MMP-9 (shown at $100 \mathrm{kDa}$ ) was reduced in parallel after GOLPH3 silencing, confirming that GOLPH3 can regulate both the expression and enzymatic activity of MMP-2 and MMP-9. Zhou et al. used the gelatin zymography assay to illustrate the effect of GOLPH3 on MMP-2 and found that GOLPH3 can affect MMP-2 activity [11], which is consistent with our findings. 
The expression levels of MMP-2 and MMP-9 are usually regulated at the transcriptional level, and a variety of cancer-related genes can influence the promoter activity of MMP2 and MMP-9 [23]. However, we found that the MMP-2 expression was not changed after silencing GOLPH3 at the mRNA level, which is consistent with the findings of Zhou et al. [11], whereas MMP-9 mRNA was expressed at low levels in A549 cells but not in L78 cells. Furthermore, we found that the proteasome inhibitor MG-132 [24] could restore the level of the MMP-2 protein in the A549 and L78 cell lines after GOLPH3 downregulation, whereas it had almost no effect on MMP-9, particularly in L78 cells. However, Zhou found that GOLPH3 affects MMP-2 activity but not its protein expression [11]. To elucidate the mechanism of GOLPH3-modulated MMP-2 protein expression, CHX was used to inhibit protein synthesis and the expression of MMP-2, and GOLPH3 was analyzed with a western blot assay. Cells treated with GOLPH3 siRNA and CHX exhibited faster degradation of MMP-2 than cells in the other groups, suggesting GOLPH3 might regulate the expression of MMP- 2 by increasing its degradation. Scott et al. found that GOLPH3 regulated the mTOR signaling pathways, which are primarily involved in protein synthesis through the S6K and 4EBP-1 proteins [25]. Therefore, we evaluated the phosphorylation of S6K after depletion of GOLPH3 by siRNA and found that GOLPH3 downregulation decreased the phosphorylation of S6K at thr359, indicating that GOLPH3 might influence the translation of proteins, including MMP-2. Altogether, these results strongly suggest that GOLPH3 might regulate the stability and affect the translation of MMP-2 in both A549 and L78 cells, thereby influencing the enzyme activity and ultimately regulating the invasion ability of the A549 and L78 cell lines.

In conclusion, our data support the feasibility of using GOLPH3 as a clinical indicator and prognostic marker for patients with NSCLC. In addition, this study shows, for the first time, that GOLPH3 plays an important role in NSCLC metastasis by regulating MMP-2 and MMP-9 at the protein level. Further investigation into the mechanism by which GOLPH3 mediates the expression of MMP-2 and MMP-9 is needed.

\section{Disclosure Statement}

The authors declare that they have no competing interests.

\section{Acknowledgments}

Ran Wang, Fen Wang and Zun-Fu Ke carried out the cell culture and western blot and PCR analysis, statistic analysis and participated in the design of the study and drafted the manuscript. Yue-Feng Wang, Wen-Hui Zhang and Shu-Hua Li were responsible for collection patients' information and IHC assay. Lian-Tang Wang was responsible for supervising and coordinating the whole work. All authors read and approved the final manuscript. This work was supported by the grants from the Foundation of China National Natural Science (No.: 81172232/H1615, 30900650/H1615 and 81372501/H1615).

\section{References}

1 Jemal A, Siegel R, Ward E, Hao Y, Xu J, Murray T, Thun MJ: Cancer statistics, 2008. CA Cancer J Clin 2008;58:71-96.

$>2$ Bunn PA Jr, Thatcher N: Systemic treatment for advanced (stage iiib/iv) non-small cell lung cancer: More treatment options; more things to consider. Introduction. Oncologist 2008;13:S1-4.

-3 Sun S, Ke Z, Wang F, Li S, Chen W, Han A, Wang Z, Shi H, Wang LT, Chen X: Overexpression of astrocyteelevated gene-1 is closely correlated with poor prognosis in human non-small cell lung cancer and mediates its metastasis through up-regulation of matrix metalloproteinase- 9 expression. Hum Pathol 2012;43:1051-1060. 
Wang et al.: High Expression of GOLPH3 in Non-Small Cell Lung Cancer Correlates with Poor Prognosis and Metastasis

4 Wu CC, Taylor RS, Lane DR, Ladinsky MS, Weisz JA, Howell KE: Gmx33: A novel family of trans-golgi proteins identified by proteomics. Traffic 2000;1:963-975.

5 Bell AW, Ward MA, Blackstock WP, Freeman HN, Choudhary JS, Lewis AP, Chotai D, Fazel A, Gushue JN, Paiement J, Palcy S, Chevet E, Lafreniere-Roula M, Solari R, Thomas DY, Rowley A, Bergeron JJ: Proteomics characterization of abundant golgi membrane proteins. J Biol Chem 2001;276:5152-5165.

6 Scott KL, Kabbarah O, Liang MC, Ivanova E, Anagnostou V, Wu J, Dhakal S, Wu M, Chen S, Feinberg T, Huang J, Saci A, Widlund HR, Fisher DE, Xiao Y, Rimm DL, Protopopov A, Wong KK, Chin L: Golph3 modulates mtor signalling and rapamycin sensitivity in cancer. Nature 2009;459:1085-1090.

7 Hua X, Yu L, Pan W, Huang X, Liao Z, Xian Q, Fang L, Shen H: Increased expression of golgi phosphoprotein-3 is associated with tumor aggressiveness and poor prognosis of prostate cancer. Diagn Pathol 2012;7:127.

8 Zeng Z, Lin H, Zhao X, Liu G, Wang X, Xu R, Chen K, Li J, Song L: Overexpression of golph3 promotes proliferation and tumorigenicity in breast cancer via suppression of the foxo1 transcription factor. Clin Cancer Res 2012;18:4059-4069.

-9 Kunigou O, Nagao H, Kawabata N, Ishidou Y, Nagano S, Maeda S, Komiya S, Setoguchi T: Role of golph3 and golph3l in the proliferation of human rhabdomyosarcoma. Oncol Rep 2011;26:1337-1342.

10 Li XY, Liu W, Chen SF, Zhang LQ Li XG, Wang LX: Expression of the golgi phosphoprotein-3 gene in human gliomas: A pilot study. J Neurooncol 2011;105:159-163.

-11 Zhou X, Zhan W, Bian W, Hua L, Shi Q, Xie S, Yang D, Li Y, Zhang X, Liu G, Yu R: Golph3 regulates the migration and invasion of glioma cells though rhoa. Biochem Biophys Res Commun 2013;433:338-344.

12 Zhang Y, Ma M, Han B: Golph3 high expression predicts poor prognosis in patients with resected non-small cell lung cancer: An immunohistochemical analysis. Tumour Biol 2014

13 Lu M, Tian Y, Yue WM, Li L, Li SH, Qi L, Hu WS, Gao C, Si LB, Tian H: Golph3, a good prognostic indicator in early-stage nsclc related to tumor angiogenesis. Asian Pac J Cancer Prev 2014;15:5793-5798.

14 Schmitz KR, Liu J, Li S, Setty TG, Wood CS, Burd CG, Ferguson KM: Golgi localization of glycosyltransferases requires a vps74p oligomer. Dev Cell 2008;14:523-534.

15 Takahashi M, Tsuda T, Ikeda Y, Honke K, Taniguchi N: Role of n-glycans in growth factor signaling. Glycoconj J 2004;20:207-212.

16 Ohtsubo K, Marth JD: Glycosylation in cellular mechanisms of health and disease. Cell 2006;126:855-867.

17 Seeliger H, Guba M, Kleespies A, Jauch KW, Bruns CJ: Role of mtor in solid tumor systems: A therapeutical target against primary tumor growth, metastases, and angiogenesis. Cancer Metastasis Rev 2007;26:611621.

18 de Visser KE, Eichten A, Coussens LM: Paradoxical roles of the immune system during cancer development. Nat Rev Cancer 2006;6:24-37.

19 Grivennikov SI, Greten FR, Karin M: Immunity, inflammation, and cancer. Cell 2010;140:883-899.

20 Nagase H, Woessner JF Jr: Matrix metalloproteinases. J Biol Chem 1999;274:21491-21494.

21 Valastyan S, Weinberg RA: Tumor metastasis: Molecular insights and evolving paradigms. Cell 2011;147:275-292.

22 Christofori G: New signals from the invasive front. Nature 2006;441:444-450.

-23 Liu L, Wu J, Ying Z, Chen B, Han A, Liang Y, Song L, Yuan J, Li J, Li M: Astrocyte elevated gene-1 upregulates matrix metalloproteinase-9 and induces human glioma invasion. Cancer Res 2012;70:3750-3759.

-24 Suzuki M, Iwasaki M, Sugio A, Hishiya A, Tanaka R, Endo T, Takayama S, Saito T: Bag3 (bcl2-associated athanogene 3) interacts with mmp-2 to positively regulate invasion by ovarian carcinoma cells. Cancer Lett 2011;303:65-71.

25 Laplante M, Sabatini DM: Mtor signaling in growth control and disease. Cell 2012;149:274-293. 